\title{
Pharmacological Manipulations of the Extinction Process of Fear-Induced Ultrasonic Vocalization in Rats
}

\author{
Takefumi KIKUSUI ${ }^{1)}$, Yukari TAKEUCHI ${ }^{1)}$ and Yuji MORI ${ }^{1)}$ \\ ${ }^{1)}$ Laboratory of Veterinary Ethology, The University of Tokyo, 1-1-1 Yayoi, Bunkyo-ku, Tokyo 113-8657, Japan
}

(Received 28 September 2000/Accepted 1 February 2001)

\begin{abstract}
The effects of pharmacological manipulation on the extinction process of fear-induced ultrasonic vocalizations (USVs), which are considered distress calls related to anxiety, were investigated. Male Wistar rats were conditioned to emit USVs by being given repeated electrical foot-shocks while in a chamber. After 10 sessions of conditioning, the animals started to emit USVs upon mere exposure to the shock chamber without being shocked. Using these animals, the extinction process of the USVs was examined. With repeated exposure to the chamber without shocks, the USVs first increased and then gradually decreased, i.e., the extinction burst was observed. Daily intraperitoneal injections of a benzodiazepine-GABA receptor agonist diazepam (DZP; $1.0 \mathrm{mg} / \mathrm{kg}$ ) or a tricyclic antidepressant clomipramine (CLM; $20 \mathrm{mg} / \mathrm{kg}$ ) inhibited this extinction burst. Moreover, CLM, but not DZP, shortened the period required for extinction as compared with the vehicle-treated animals. Following the extinction phase, the emission of USVs was enhanced by the cessation of both drug treatments. These results suggest that CLM would be useful for reducing anxiety-related behaviors in the extinction process, as long as withdrawal symptoms after long-term drug treatments are taken into consideration.

KEY WORDS: clomipramine, diazepam, extinction, ultrasonic, vocalization.
\end{abstract}

J. Vet. Med. Sci. 63(6): 591-595, 2001

Excessive anxiety is one of the largest affective disorders in humans, and a similar symptom has been observed in dogs, namely "separation anxiety" which is defined as excessive vocalization, inappropriate elimination and destruction associated with the owner's absence [3, 21, 34]. Behavioral modification, such as desensitization and counter-conditioning, are the most important and effective measures for solving these behavioral problems in dogs [3, $21,34]$, as in human clinics. Behavioral modification is, however, sometimes accompanied by an increase in anxiety or aggression [20]. Supplementary drug treatment is also efficacious against behavioral symptoms in dogs [18], although the interaction between behavioral modification and drug treatment has not been well established.

Rodents emit ultrasonic vocalizations (USVs) under adverse circumstances, such as being separated from littermates [1, 24], during agonistic confrontations [22, 30, 35, $38]$, and being exposed to foot shocks $[9,36]$ or to acoustic stimuli [16]. It has been demonstrated that the USVs induced by foot shocks are mediated by corticotropinereleasing factor [17] and are attenuated by anti-anxiety drugs in adult rats $[9,36]$, and thus the USVs have been regarded as a good index for anxiety status in this animal model $[9,22]$. This model possesses the following similarities to separation anxiety in dogs: distress calls, anticipative anxiety-related behaviors and active avoidance behaviors that are based on contextual memory.

In this study, we investigated the interaction between behavioral therapy and drug treatments by using the rat USVs model. Namely, the effects of daily intraperitoneal injections of a benzodiazepine-GABA receptor agonist diazepam (DZP) and tricyclic antidepressant clomipramine (CLM) on the extinction phases of conditioned USVs were investigated. In addition, drug withdrawal symptoms were examined by assessing the USVs following the chronic drug treatments during the extinction phase.

\section{MATERIALS AND METHODS}

Animals: Male adult Wistar rats were purchased from Nippon Crea Inc. (Yokohama, Japan). At the beginning of training, the animals were 9 to 10 weeks old. They were housed 3-5 animals per cage in a room with a 12:12 hr light/ dark cycle (light on from 8 a.m. to 8 p.m.), under an environment kept at a constant temperature $\left(24 \pm 1^{\circ} \mathrm{C}\right)$ and humidity $(45 \pm 5 \%)$. Food and water were provided ad libitum. The experimental procedures followed the guidelines of "Policies Governing The Use of Live Vertebrate Animals" by University of Tokyo, based on "The public health Service Policy on Human Care and Use of Laboratory Animals" by Awardee Institution (revised 1985), and "The National Institutes of Health guide for The Care and Use of Laboratory Animals" (revised 1985). All experiments were conducted from 9 a.m. to 6 p.m.

Apparatus and procedure: The experimentally naive rats were put into a small chamber $(28 \mathrm{~cm}$ in width, $18 \mathrm{~cm}$ in depth, $26 \mathrm{~cm}$ in height) with electrifiable grids. The small chamber was placed in a soundproof chamber $(60 \mathrm{~cm}$ in width, $70 \mathrm{~cm}$ in depth, $60 \mathrm{~cm}$ in height; Muromachi Kikai, Co., Tokyo, Japan) equipped with a microphone (Type 4116, Aco Co., Tokyo, Japan) and a CCD camera (IK-M43 $\mathrm{H} 47$, Toshiba, Tokyo, Japan) in the center of the ceiling. The microphone was connected to an amplifier and a bandpass filter that selected from $10 \mathrm{kHz}$ to $100 \mathrm{kHz}$. The transformed signals were visualized and recorded by a real-time spectrum analyzer (3650, Sony Tektronix, Tokyo, Japan). 
The CCD camera was connected to a video monitor for observing the animals' behaviors.

Conditioning: Each session, consisting of 3 parts, was conducted once a day and continued for more than 10 days. The first part of each session was a 10-min pre-shock period (just exposing the animals to the shock chamber), the second part was a 10-min shock period, and the third part was a 5min post-shock period. During the shock period, the animals received intermittent inescapable electrical shocks (1.25 mA) according to the following schedule: one series of 3 shocks (duration $0.2 \mathrm{sec}, 2$-sec interval, duration $2 \mathrm{sec}, 2$ sec interval, duration $0.2 \mathrm{sec}$.) was repeated 3 times. The interval between each series of 3 shocks was randomized from $3 \mathrm{~min}$ to $7 \mathrm{~min}$. When the rat squeaked or emitted a USV, the delivery of foot shocks was interrupted so that the animal would not form an association between shocks and vocalizations.

USVs were measured at the pre-shock period from $8 \mathrm{~min}$ to $10 \mathrm{~min}$ after the rat was exposed to the chamber (PRE), and at the post-shock period from $1 \mathrm{~min}$ to $2 \mathrm{~min}$ (POST 1 ) and from 4 min to 5 min (POST 2) after the shock period. USVs were spectrum-analyzed and recorded in a real-time spectrum analyzer. The amplitudes of the signals in respective measures were averaged for each frequency. The average amplitude was then summed from $20 \mathrm{kHz}$ to $100 \mathrm{kHz}$. The data are therefore combined products of the intensity and duration of USVs [17]. The criterion for determining whether a rat had acquired conditioning was total amplitude of the USV in the PRE period of more than $15 \mathrm{~dB} \bullet \mathrm{sec}$.

Extinction: The animals that fulfilled the criterion were thereafter used for the extinction tests. They were placed into the same chamber that had been used for the conditioning, and then they received a small electrical shock $(0.1 \mathrm{~mA}$, $0.2 \mathrm{sec}$ ), which had never induced USVs in experimentally naïve rats, for the cue signal of each session start. One session was 15-min without any shock and was repeated until the USVs disappeared. The pharmacological manipulation was conducted before each session. The USVs from 5 to 6 , 10 to 11 , and 15 to $16 \mathrm{~min}$ (total $3 \mathrm{~min}$ ) after the cue shocks were measured as described above. The USVs were re-calculated as the percentage of the amplitude of just after conditioning. Extinction was defined as complete when the USVs were less than $15 \%$ in two consecutive sessions. In the next session, the animals were tested for a no-drug session, in which they performed the same session as the extinction session without drug manipulations in order to allow us to evaluate the drug withdrawal effects on the extinction processes.

Drugs: Diazepam (DZP; Sigma, St. Louis, MO, U.S.A.) and clomipramine hydroxycloride (CLM; Sigma, St. Louis, MO, U.S.A.) were dissolved in $0.5 \%$ tragacanth saline. Due to different protocols of drug administration, the DZP and CLM groups were provided with their own control groups respectively. DZP $1 \mathrm{mg} / \mathrm{kg}(\mathrm{n}=6)$, or vehicle $(\mathrm{n}=6)$ was injected intraperitoneally $30 \mathrm{~min}$ before each session. CLM $20 \mathrm{mg} / \mathrm{kg}(\mathrm{n}=6)$ or vehicle $(\mathrm{n}=6)$ was injected intraperitoneally $45 \mathrm{~min}$ before each session. The doses of DZP and
CLM were selected according to the previous reports in which these drugs showed decreasing effects on the USVs in rats $[9,38]$

Data analysis: Data analyses were performed with StatView + Graphics 4.1J (Abacus Concepts, Inc., Berkeley, CA, U.S.A.). The significance level for all statistical tests was set at $\mathrm{p}=0.05$. A one-way ANOVA was performed to compare the number of sessions that were needed for extinction, and to compare the USVs in the withdrawal test.

\section{RESULTS}

The animals emitted regular USVs with a frequency range of 21 to $23 \mathrm{kHz}$ and durations of 0.9 to $1.3 \mathrm{sec}$ after 10 conditioning sessions. Changes of USVs in the control and drug-treated groups during the extinction process are shown in Fig. 1. In the extinction phase, the USVs first increased and thereafter gradually decreased in 5 out of 12 control animals, i.e., an extinction burst was observed. Of the 7 remaining control animals, 3 showed similar levels and 4 showed decreased levels of the USVs in the early phase of the extinction sessions. By contrast, none of the DZP- or CLM-treated animals showed the extinction burst. DZP-
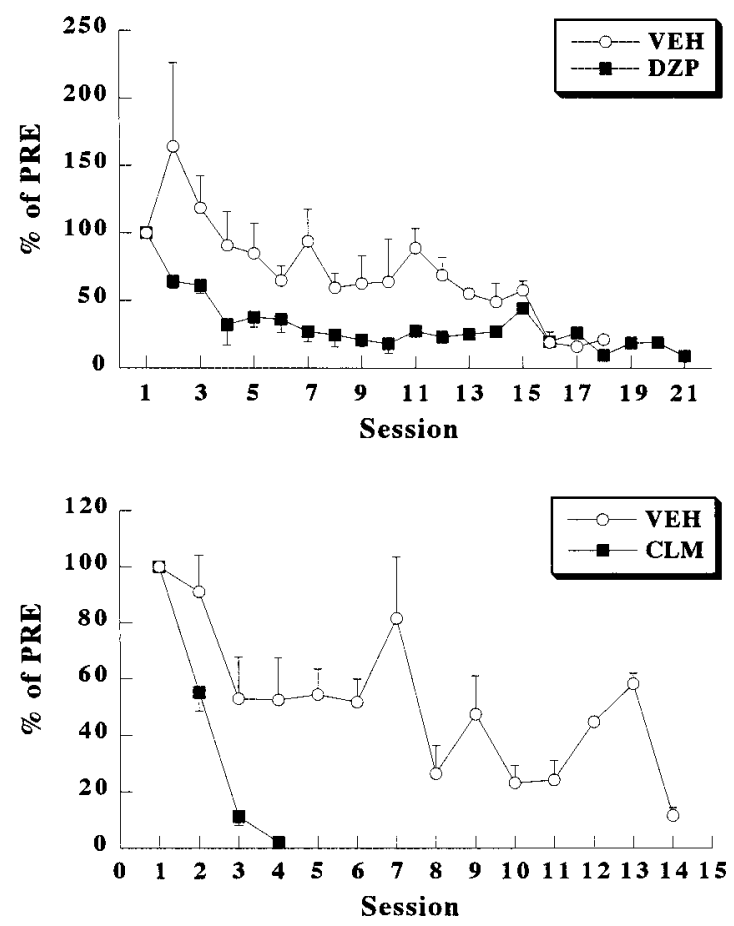

Fig. 1. The changes of the USVs in the extinction phase. The USVs once increased and thereafter gradually decreased in the half of the control animals. All of the DZP or CLM treated animals did not show any extinction burst. The USVs in the animals treated with CLM, but not DZP, decreased rapidly. The number of animals for each point decreased from 10 to 2, because the animals that fulfilled the extinction criteria were excluded from the data in sequence. 

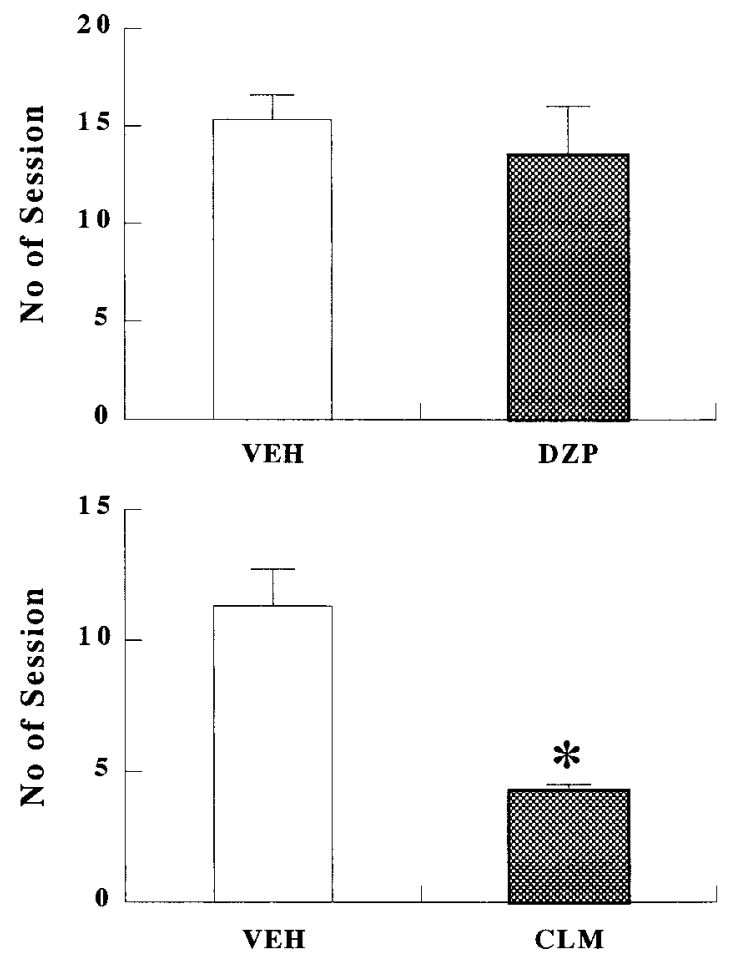

Fig. 2. The number of the sessions needed for extinction. The animals treated with CLM, but not DZP, fulfilled the criteria for extinction faster than the vehicle treated animals. (* $\mathrm{p}<0.005$, One-way ANOVA).

treated rats emitted fewer USVs compared with vehicletreated animals, and the USVs decreased gradually (Fig. 1). On the other hand, the USVs in the CLM-treated animals decreased rapidly (Fig. 1). The number of sessions needed for extinction was $13.5 \pm 2.8$ for DZP, $15.3 \pm 3.4$ for vehicle; and $4.5 \pm 0.2$ for CLM, $11.3 \pm 1.5$ for vehicle (Fig. 2). A one-way ANOVA revealed that CLM, but not DZP, reduced the number of sessions needed for extinction (Fig. 2, DZP, $\mathrm{F} 1,10=0.34, \mathrm{p}=0.57$; CLM, F1,10=19.77, $\mathrm{p}<0.005)$. The response in USVs to the interruption of drug treatment is shown in Fig. 3. DZP- and CLM-treated animals emitted USVs more than vehicle-treated animals (DZP, F1, $10=11.80, \mathrm{p}<0.01 ; \mathrm{CLM}, \mathrm{F} 1,10=6.1, \mathrm{p}<0.05)$.

\section{DISCUSSION}

The animals were conditioned to emit USVs with repeated exposure to electrical shocks, and they started to emit USVs of $20-23 \mathrm{kHz}$ with a duration of 0.9 to $1.3 \mathrm{sec}$ when they were brought into the shock chamber without any shocks, indicating that contextual fear conditioning was acquired in the tested animals, as we previously described [17]. Distress USV calls under anxiety-induced situations are considered to be one of the active avoidance behaviors, because the emission of the USVs can ameliorate undesirable events, such as long-term isolation from littermates in
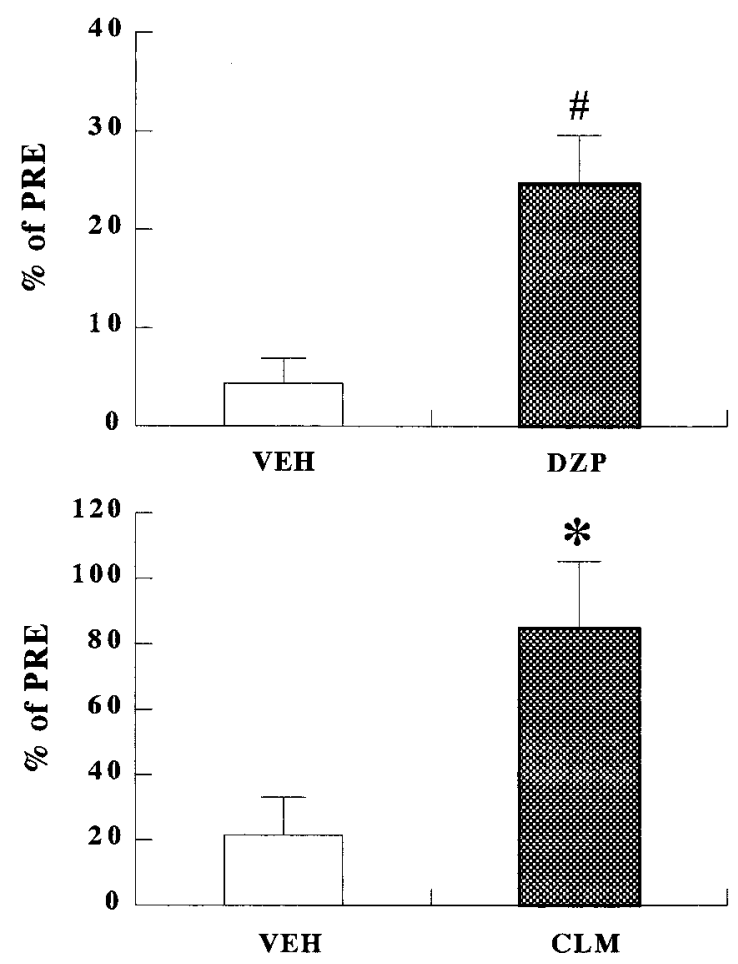

Fig. 3. The USVs in the absence of drug treatment following the chronic pharmacological manipulations. The DZP and CLM treated animals emitted more USVs compared with vehicle treated animals (\# p<0.01, * p<0.05; One-way ANOVA).

pups [1, 24] and hard attacks from intruders in social confrontation models [30]. Thus, the USVs observed in the preshock period are considered to be distress calls in anticipation of electrical shocks (anxiety-related behaviors) and also an active avoidance behavior based on contextual memory.

With repeated exposure to the same context without shocks, the USVs once increased and thereafter gradually decreased, i.e., an extinction burst was observed. It was reported that extinction bursts occurred before the behavior disappeared in rats $[4,8]$ and humans $[20,39]$. Both DZP and CLM inhibited extinction bursts in the present study, and this result is consistent with the previous findings that DZP decreased the USVs induced by foot shocks in rats [9, 38]. Contrary to our results, another tricyclic antidepressant, imipramine, was reported to have little effect on the USVs [9]. This may be due to the fact that CLM is more selective to 5-hydroxy triptamine $(5-\mathrm{HT})$ reuptake inhibition as compared to imipramine [31,37], as selective 5-HT reuptake inhibitors decreased USVs in the same experimental model [9]. The 5-HTergic systems have an important role in modulation of the USVs, because 5-HT-related anxiolytics such as selective 5-HT 1A receptor agonist 8-OH DPAT and buspirone decreased the USV[s] induced by foot shocks [9], and also selective 5-HT 1A receptor agonist 8OH DPAT and flesinoxan, as well as selective 5-HT 1B receptor agonist CP-94,253, reduced the USVs in mater- 
nally separated mouse pups [11]. We previously reported that the central corticotropin releasing factor (CRF) systems play an important role in the emission of USVs, especially in the retrieval process of fear-related memory [17]. The amygdaloid CRF neurons innervate their fibers to the dorsal and medial raphe nuclei $[19,29]$, in which 5-HT neurons highly exist, and modulate 5-HT neuron activity [19, 26]. The neural circuit including the amygdaloid CRF system and GABAergic system, and the 5-HT system in the raphe nucleus, may thus be responsible for coding as well as expression of distress USVs $[11,17]$.

In both DZP and CLM tests following the extinction phase, the USV response was exaggerated in the absence of drug treatment. This may be explained by the fact that both drugs impair the extinction of fear-related behavior, as previously reported $[27,28]$. Recent studies showed, however, that the effects of these drugs were state-dependent $[4,6]$. It has often been convenient to assume that the gradual loss of fear performance that occurs during the extinction process is due to a weakening of the association between original conditioning stimuli (CS) and unconditioning stimuli (US) [27, 28]. However, there is good evidence that the original association can survive even after a large number of extinction trials in both laboratory animals [4] and clinical cases [5, 39], i.e., relapses of fear behavior were often observed. Recent studies suggest that memories corresponding to both conditioning and extinction may be stored and become available as long-term memory; performance after extinction may thus be determined by which memory is accessed or retrieved by the corresponding retrieval cues $[4,6]$. Supporting this hypothesis are the findings that amnesiac drugs, such as acetylcholine muscarinic receptor antagonist scopolamine [25, 32], GABA A receptor agonists DZP [6, 12, 14, 15] and muscimol [13, 23], and NMDA receptor antagonist D,L-2-amino-5-phosphonovaleric acid (AP5) [10] impair both the acquisition and extinction of context fear memory. Therefore, learning new information, i.e., unpairing of CSUS, which occurs under the influence of a drug, may not be transferred to an undrugged condition; this is referred to as state-dependent retention, and is often outside the original drug state $[2,5-7,33]$. In the present study, the rats could have learned the extinction as a new association between the drugged state and the context of no-shock. Therefore the drug may have been perceived as a "cue" of no-shock. However, they did not express extinction behavior outside the drugged state, as Bouton et al. showed that benzodiazepine has state-dependent extinction [6]. Similar statedependent effects of DZP were reported in human clinics [5, 14, 15]. Further investigation is needed for testing this hypothesis.

CLM, but not DZP, shortened the period required for extinction as compared with the vehicle-treated rats. Clinically, CLM has similar positive effects on behavioral modification in separation anxiety in dogs [18]. This may be a suggestive report as it showed a similar effect of CLM on an anxiety-related behavior in rodents. However, the effects of CLM appear to be temporal, as the USVs markedly increased in the absence of drug administration after the extinction. It is worth prescribing CLM for separation anxiety in dogs, because the effects of CLM in inhibiting the extinction burst and shortening the period of extinction may be beneficial to dog owners. The owners sometimes feel disappointed when they see an increase of anxiety-related behavior in the early phase of behavioral therapy, i.e., the extinction burst. In this phase, if CLM can decrease the anxiety-induced behavior, the owner may become more confident that the behavioral therapy is going to be successful and they will comply with the therapy. Continuous behavioral therapy is strongly needed for treatment of separation anxiety, which sometimes takes up to several months [3, 21]. From this aspect, supplementary drug treatment to the behavioral therapy is useful for separation anxiety in dogs, even if cessation of the drug may cause some withdrawal symptoms.

In conclusion, it has been demonstrated that DZP and CLM inhibit the extinction burst in the context fear-conditioning model in rats, and that CLM, but not DZP, shortens the period required for extinction. Although cessation of either drug may cause withdrawal symptoms, drug treatments appear useful for promoting the extinction of anxietyrelated behaviors, such as separation anxiety in dogs, because decreasing the extinction burst would be largely advantageous for behavioral modification programs. In addition, it is suggested that an insight into state-dependent fear extinction would provide a way to understand the mechanism of relapse of anxiety after clinical therapy, in which clients who are prescribed DZP or CLM for their dogs in combination with an "exposure" therapy may run a risk of renewing fear response once the drug is discontinued.

ACKNOWLEDGMENT. The present study was financially supported by CREST of the Japan Science and Technology Agency.

\section{REFERENCES}

1. Allin, J. T. and Banks, E. M. 1972. Functional aspects of ultrasound production by infant albino rats (Rattus norvegicus). Anim. Behav. 20: 175-185.

2. Barry, H. D., Etheredge, E. E. and Miller, N. E. 1965. Counterconditioning and extinction of fear fail to transfer from amobarbital to nondrug state. Psychopharmacologia 8: 150156.

3. Borchelt, P. L. and Voith, V. L. 1982. Diagnosis and treatment of separation-related behavior problems in dogs. Vet. Clin. North Am. Small Anim. Pract. 12: 625-635.

4. Bouton, M. E. 1988. Context and ambiguity in the extinction of emotional learning: implications for exposure therapy. Behav. Res. Ther. 26: 137-149.

5. Bouton, M. E. 2000. A learning theory perspective on lapse, relapse, and the maintenance of behavior change. Health Psychol. 19: 57-63.

6. Bouton, M. E., Kenney, F. A. and Rosengard, C. 1990. Statedependent fear extinction with two benzodiazepine tranquilizers. Behav. Neurosci. 104: 44-55.

7. Cunningham, C. L. 1979. Alcohol as a cue for extinction: State 
dependency procedure by conditioned inhibition. Anim. Learn. Behav. 7: 45-52.

8. Davis, M. and Astrachan, D. I. 1978. Conditioned fear and startle magnitude: effects of different footshock or backshock intensities used in training. J. Exp. Psychol. Anim. Behav. Proc. 4: 95-103.

9. De Vry, J., Benz, U., Schreiber, R. and Traber, J. 1993. Shockinduced ultrasonic vocalization in young adult rats: a model for testing putative anti-anxiety drugs. Eur. J. Pharmacol. 249: 331-339.

10. Falls, W. A., Miserendino, M. J. and Davis, M. 1992. Extinction of fear-potentiated startle: blockade by infusion of an NMDA antagonist into the amygdala. J. Neurosci. 12: 854863.

11. Fish, E. W., Sekinda, M., Ferrari, P. F., Dirks, A. and Miczek, K. A. 2000. Distress vocalizations in maternally separated mouse pups: Modification via 5-HT1A, 5-HT1B and GABAA receptors. Psychopharmacology 149: 277-285.

12. Grant, K. A. and Johanson, C. E. 1987. Diazepam self-administration and resistance to extinction. Pharmacol. Biochem. Behav. 28: 81-86.

13. Helmstetter, F. J. and Bellgowan, P. S. 1994. Effects of muscimol applied to the basolateral amygdala on acquisition and expression of contextual fear conditioning in rats. Behav. Neurosc. 108: 1005-1009.

14. Jensen, H. H., Hutchings, B. and Poulsen, J. C. 1989. Conditioned emotional responding under diazepam: a psychophysiological study of state dependent learning. Psychopharmacology 98: 392-397.

15. Jensen, H. H. and Poulsen, J. C. 1982. Amnesic effects of diazepam: "drug dependence" explained by state-dependent learning. Scand. J. Psychol. 23: 107-111.

16. Kaltwasser, M. T. 1990. Startle-inducing acoustic stimuli evoke ultrasonic vocalization in the rat. Physiol. Behav. 48: 13-17.

17. Kikusui, T., Takeuchi, Y. and Mori, Y. 2000. Involvement of cortcotropin-releasing factor in the retrieval process of fearconditioned ultrasonic vocalization in rats. Physiol. Behav. 71: 323-328.

18. King, J. N., Simpson, B. S., Overall, K. L., Appelby, D., Pageat, P., Ross, C., Chaurand, J. P., Heath, S., Beata, C., Weiss, A. B., Muller, G., Paris, T., Bataille, B. G., Parker, J., Petit, S. and Wren, J. 2000. Treatment of separation-related anxiety in dogs with clomipramine: results from a prospective, randomized, double-blind, placebo-controlled, parallel-group, multicenter clinical trial. Appl. Anim. Behav. Sci. 67: 255-275.

19. Kirby, L. G., Rice, K. C. and Valentino, R. J. 2000. Effects of corticotropin-releasing factor on neuronal activity in the serotonergic dorsal raphe nucleus. Neuropsychopharmacology 22: $148-162$.

20. Lerman, D. C., Iwata, B. A. and Wallace, M. D. 1999. Side effects of extinction: prevalence of bursting and aggression during the treatment of self-injurious behavior. J. Appl. Behav. Anal. 32: 1-8.

21. McCrave, E. A. 1991. Diagnostic criteria for separation anxiety in the dog. Vet. Clin. North Am. Small Anim. Pract. 21: 247255.

22. Miczek, K. A., Tornatzky, W. and ViVian, J. A. 1991. Ethology and neuropharmacology: rodent ultrasounds. pp. 409-427 In Animal Models in Psychopharmacology (Olivier, B., Moss, J. and Slangen, J.L. eds.), Birkhauser, Basel.
23. Muller, J., Coordimas, K. P., Fridel, Z. and LeDoux, J. E. 1997. Functional inactivation of the lateral and basal nuclei of the amygdala by muscimol infusion prevents fear conditioning to an explicit conditioned stimulus and to contexual stimuli. Behav. Neurosci. 111: 683-691.

24. Noirot, E. 1970. Selective priming of maternal responses by auditory and olfactory cues from mouse pups. Dev. Psychobiol. 2: 273-276.

25. Prado-Alcala, R. A., Haiek, M., Rivas, S., Roldan-Roldan, G. and Quirarte, G. L. 1994. Reversal of extinction by scopolamine. Physiol. Behav. 56: 27-30.

26. Price, M. L., Curtis, A. L., Kirby, L. G., Valentino, R. J. and Lucki, I. 1998. Effects of corticotropin-releasing factor on brain serotonergic activity. Neuropsychopharmacology 18 : 492-502.

27. Rescorla, R. A. 1973. Effect of US habituation following conditioning. J. Comp. Physiol. Psychol. 82: 137-143.

28. Rescorla, R. A. 1976. Stimulus generalization: some predictions from a model of Pavlovian conditioning. J. Exp. Psychol. Anim. Behav. Process 2: 88-96.

29. Ruggiero, D. A., Underwood, M. D., Rice, P. M., Mann, J. J. and Arango, V. 1999. Corticotropic-releasing hormone and serotonin interact in the human brainstem: behavioral implications. Neuroscience 91: 1343-1354.

30. Sales, G. D. 1972. Ultrasound and agressive behaviour in rats and other small mammals. Anim. Behav. 20: 88-100.

31. Sangdee, C. and Franz, D. N. 1979. Enhancement of central norepinephrine and 5-hydroxytryptamine transmission by tricyclic antidepressants. A comparison. Psychopharmacology 62: 9-16.

32. Scavio, M. J., Clift, P. S. and Wills, J. C. 1992. Posttraining effects of amphetamine, chlorpromazine, ketamine, and scopolamine on the acquisition and extinction of the rabbit's conditioned nictitating membrane response. Behav. Neurosci. 106: 900-908.

33. Stain, L. and Berger, B. D. 1969. Paradoxical fear-increasing effects of tranquilizers: Evidence of repression of memory in the rats. Science 166: 253-256.

34. Takeuchi, Y., Houpt, K. A. and Scarlett, J. M. 2000. Effectiveness of treatment for canine separation anxiety. J. Am. Vet. Med. Assoc. 217: 342-345.

35. Thomas, D. A., Takahashi, L. K. and Barfield, R. J. 1983. Analysis of ultrasonic vocalizations emitted by intruders during aggressive encounters among rats (Rattus norvegicus). $J$. Comp. Psychol. 97: 201-6.

36. Van der Poel, A. M., Noach, E. J. and Miczek, K. A. 1989. Temporal patterning of ultrasonic distress calls in the adult rat: effects of morphine and benzodiazepines. Psychopharmacology 97: 147-148.

37. van Wijk, M., Meisch, J. J. and Korf, J. 1977. Metabolism of 5hydroxytryptamine and levels of tricyclic antidepressant drugs in rat brain after acute and chronic treatment. Psychopharmacology 55: 217-223.

38. Vivian, J. A. and Miczek, K. A. 1993. Diazepam and gepirone selectively attenuate either $20-32$ or $32-64 \mathrm{kHz}$ ultrasonic vocalizations during aggressive encounters. Psychopharmacology 112: 66-73.

39. Vollmer, T. R., Ringdahl, J. E., Roane, H. S. and Marcus, B. A. 1997. Negative side effects of noncontingent reinforcement. $J$. Appl. Behav. Anal. 30: 161-164. 